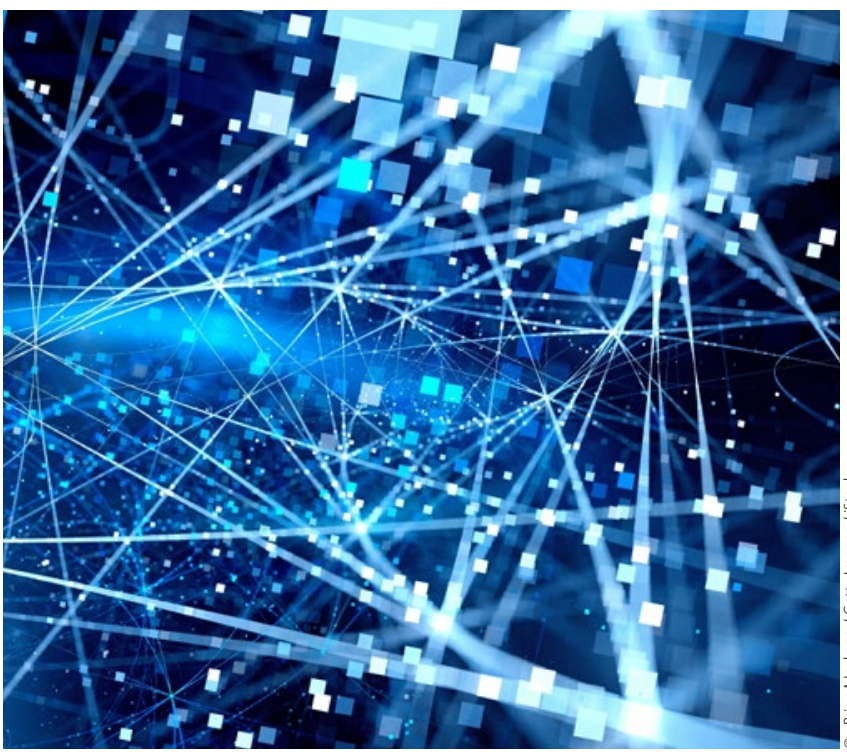

Ziel ist ein digital vernetztes Gesundheitssystem

\section{Medizininformatik- Initiative}

Nach dem Willen der Bundesregierung sollen Ärzte und Zahnärzte bei der Behandlung von Patienten per Knopfdruck auf eine Vielzahl von Daten und wissenschaftlichen Erkenntnissen zugreifen können. Deshalb hat das Bundesministerium für Bildung und Forschung (BMBF) eine Medizininformatik-Initiative gestartet, die mit mehr als 150 Millionen Euro gefördert wird. Eine digital vernetzte Medizin soll eine schnellere Diagnostik und wirkungsvollere Therapien ermöglichen.

„Es werden in Deutschland schon sehr viele Daten erfasst, aber sie werden noch nicht optimal genutzt", sagte die Bundesforschungsministerin Johanna Wanka vergangenen Montag bei einer Pressekonferenz in Berlin. Vor allem im Bereich von seltenen Erkrankungen gebe es noch sehr viel Verbesserungspotenzial. Ziel sei es, die wachsenden Datenschätze - von Röntgenbildern bis hin zu Erbgut-Analysen - in einer nationalen Infrastruktur zu verknüpfen.

Das BMBF hat mit Hilfe eines internationalen Expertenkreises entschieden, vier Konsortien, bestehend aus 17 deutschen Universitätskliniken und rund 40 weiteren Partnern, in die vierjährige Aufbau- und Vernetzungsphase aufzunehmen. Ab 2018 werden die Konsortien dann sogenannte Datenintegrationszentren aufbauen, über die sie sich vernetzen und Daten austauschen können.

„Wir wollen in den nächsten vier Jahren einen entscheidenden Schritt vorankommen, um das Projekt dann bundesweit aufzurollen“, kündigte Wanka an. „Die elektronische Patientenakte ist perspektivisch das, was wir wollen."

Angst vor Datenmissbrauch sei unbegründet, sagte die Ministerin. Deutsche Datenschutz-Standards, die sehr streng seien, würden selbstverständlich eingehalten.
Start-ups in der Gesundheitswirtschaft

\section{Schöne neue Welt der Digitalisierung}

Die CDU hat in der nun vergangenen Legislaturperiode eine „E-Health-Strategie“ für Deutschland entwickelt. Zwölf Punkte umfasst das Programm für ein „digitalisiertes Gesundheitswesen“. Dabei geht es hauptsächlich um Vernetzung, bessere medizinische Versorgung und neue Therapiemöglichkeiten. Aus dem CDU-geführten Bundesgesundheitsministerium kam das E-Health-Gesetz dazu, das sich nahtlos einreihte. Auch die SPD entdeckte das Thema für sich. Aus dem sozialdemokratisch geführten Bundeswirtschaftsministerium (BMG) kam dann im Frühjahr dieses Jahres ein Papier mit großem Aufschlag ans Tageslicht, in dem es beim Thema E-Health nicht um Patienten oder Ärzte ging, sondern um die Gesundheitswirtschaft, die als einer der größten deutschen Wirtschaftssektoren ausgemacht wurde. Das Tempo der Digitalisierung allerdings lasse dort „zu wünschen übrig", deshalb will das Wirtschaftsministerium ein Programm auflegen, um „die Digitalisierung der Gesundheitswirtschaft zu beschleunigen und innovative Start-ups auf diesem Markt zu unterstützen“. Bundeseinheitliche, transparente und verbindliche Datenschutzregelungen sollen beim Umgang mit Gesundheitsdaten natürlich umgesetzt und eingehalten werden.

Ansonsten will das Ministerium die digitalen Neugründer vor allem beim Zugang in die Regelversorgung durch die gesetzlichen Krankenkassen unterstützen, der eine zentrale Hürde für innovative Produkte der Gesundheitswirtschaft darstelle. Bemängelt wird vom Ministerium, dass im Bereich der Gesundheitswirtschaft der Zugang zu Risikokapital für Gründer extrem schwierig sei. Auch dort sollten Hürden beseitigt werden. Und ein weiterer Big-Point ist Big Data. Dass viele Daten, die heute von Patienten selbst erhoben würden, mit Daten aus dem professionellen medizinischen Bereich nicht verknüpft werden könnten, hemme die Entwicklung für innovative Geschäftsmodelle auf Basis von Big Data. Dass die neun Punkte, die das Wirtschaftsministerium nicht ganz konform gehen mit den Vorstellungen aus dem BMG ficht die Politiker derzeit nicht an. Digitalisierung in Zeiten des Wahlkampfes ist eben auch ein großes Thema.

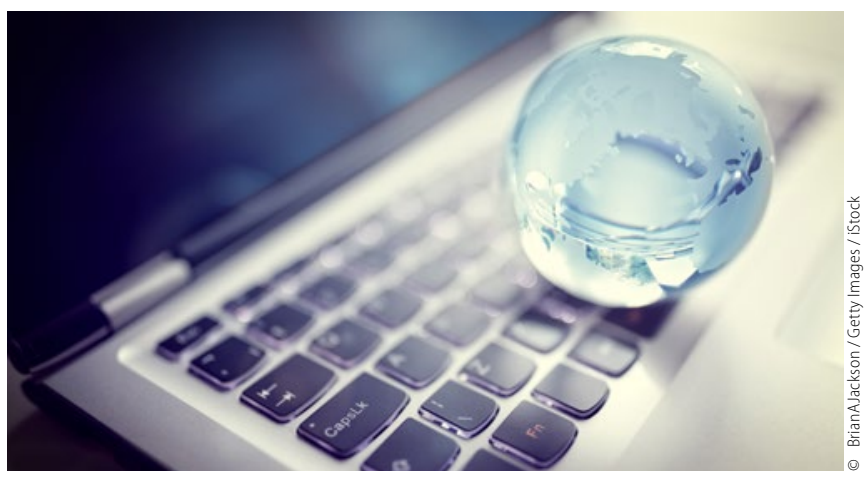

